\title{
Tourisme et patrimoine au Mali. Destruction des valeurs anciennes ou valorisation concertée?
}

\author{
Walter E.A. van Beek, Leiden et Utrecht, Philippe \\ Lemineur, Bruxelles, Olivier Walther, Luxembourg
}

\section{Des sites maliens à l'épreuve du tourisme}

Le classement au Patrimoine de l'Humanité de l'ancienne ville de Djenné et de Tombouctou, des Falaises de Bandiagara-Pays Dogon et du tombeau des Askia à Gao témoignent de la volonté du Mali de valoriser la richesse des lieux reconnus pour leur «valeur universelle exceptionnelle» (UNESCO 2006). Ces initiatives s'inscrivent toutefois dans un environnement fortement marqué par la pauvreté et ne peuvent généralement se limiter à la préservation architecturale du patrimoine sans également prendre en considération les conditions socio-économiques des bénéficiaires (OuAlLet 2002). Un des moyens de concilier la valorisation des sites maliens et les contraintes qui s'exercent sur les populations est d'ouvrir ces lieux d'exception au tourisme, en bénéficiant de l'actuel engouement international pour le Mali. Toutefois, la mise en tourisme du patrimoine reste un sujet de controverse qui divise les partisans d'une ouverture généralisée des destinations culturelles, favorables au développement des opportunités économiques, et leurs adversaires, qui s'inquiètent des répercussions que les activités touristiques pourraient entraîner sur les modes de vie «traditionnels» et qui privilégient la stricte conservation du patrimoine culturel et construit.

C'est avec l'ambition de discuter de cette controverse qu'est conduite l'étude des initiatives de conservation entreprises au Mali, en traitant plus spécifiquement des Falaises de Bandiagara-Pays Dogon et de la vieille ville de Djenné qui ont fait l'objet de programmes de protection, ainsi que des Monts Hombori qui n'ont bénéficié d'aucune mesure (carte 1). L'hypothèse de cet article est de considérer que les positions précédemment exposées ne sont pas nécessairement irréconciliables, dans la mesure où sont également prises en compte les stratégies endogènes des acteurs du tourisme. Il est par conséquent possible d'imaginer que la conservation soit basée sur le principe d'un cercle vertueux dans lequel la protection offerte aux sites renforce leur attractivité touristique et permettent, en retour, une amélioration des conditions de vie des populations (JHA 2005). C'est pourquoi une attention toute particulière est accordée aux principales dynamiques locales, en focalisant l'intérêt sur la spécificité de la «bulle touristique» qui permet aux acteurs maliens de concilier le tourisme en parallèle de leurs activités sociales habituelles et sur les programmes qui se sont donnés pour objectifs de restaurer le patrimoine malien. Le principe méthodologique qui soustend ces approches différentes est donc de considérer le tourisme, non pas comme un phénomène exogène qui produirait nécessairement un «impact» évaluable en termes positifs ou négatifs, mais de tenir compte des stratégies internes qui concourent à différencier les espaces et les cultures en attribuant une certaine autonomie aux acteurs locaux.

Les résultats présentés forment la synthèse des travaux développés par les trois auteurs depuis le début des années 1990 dans le cadre de leurs recherches en anthropologie, architecture et géographie: (1) Etudes menées dans la Falaise de Bandiagara selon les principes de l'observation participante par VAN BEEK (1991, 2001, 2003, 2005). (2) Initiatives de restauration architecturale du patrimoine malien entreprises dans le cadre du Projet de Réhabilitation et de Conservation de l'Architecture de Djenné (1996-2003), du Projet de sauvegarde du Patrimoine culturel au Mali (2004-2007) et de la Mission pour la documentation des typologies architecturales du Pays Dogon (2001) mis en œuvre par les Pays-Bas et le Ministère de la Culture du Mali (Dijk, Lemineur \& Schuts 2004). Ces initiatives supposent un relevé précis des différentes composantes architecturales dignes d'être préservées et permettent de déboucher sur une réhabilitation du patrimoine construit. (3) Missions ponctuelles entreprises depuis 2002 par les universités de Neuchâtel et de Lausanne et leurs partenaires maliens au travers du Projet de monitoring écologique Hombori et menées sous forme d'enquêtes sociologiques dans l'ensemble des villages des Monts (WaLTHER 2006).

\section{Les initiatives de restauration}

Au Mali, la mise en œuvre des initiatives de conservation et de mise en valeur du patrimoine est confiée aux Missions culturelles, chargées depuis 1993 des mesures juridiques, techniques, administratives et financières adéquates ainsi que de la mise en œuvre des activités de formation (CISsÉ 1995). Ces missions ont pour tâches d'assister le Ministre de la Culture dans les sites inscrits sur la Liste du Patrimoine Mondial. Elles veillent en particulier au respect des règles de la préservation du patrimoine et sensibilisent les populations locales à la gestion de leur patrimoine. Cette mis- 


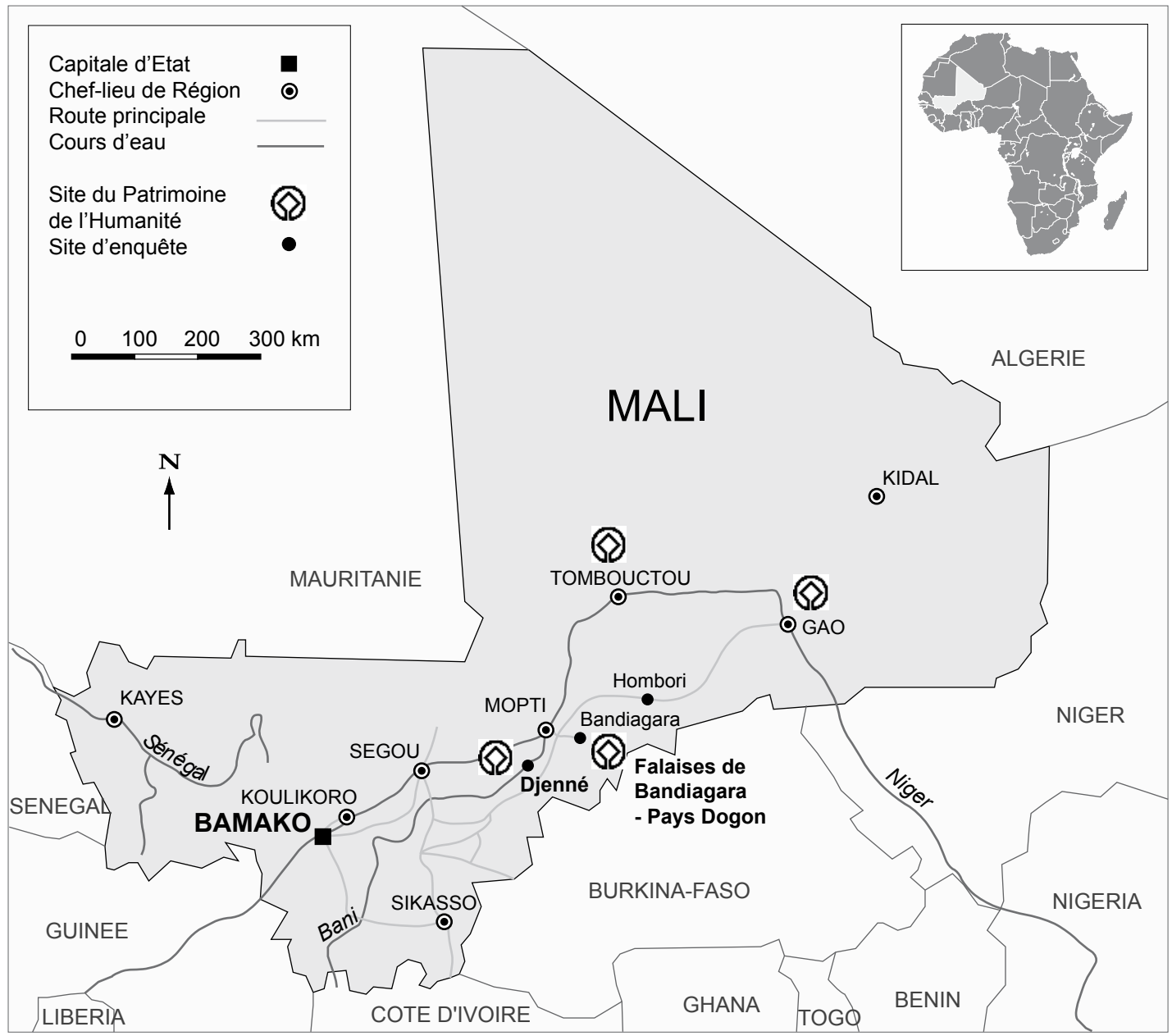

Carte 1: Localisation de Djenné, des Falaises de Bandiagara-Pays Dogon et de Hombori au Mali Location of Djenné, the Bandiagara Escarpment in Dogon country and Hombori in Mali Geographische Lage von Djenné und der Bandiagara-Felswand im Gebiet der Dogon sowie von Hombori in Mali Source: WALTHER 2006; cartography: O. WALTHER

sion n'est pas toujours facilitée par le fait qu'il existe parfois une profonde divergence entre les principes internationaux de restauration des monuments et les normes esthétiques locales et l'utilisation de matériaux comme le ciment ou la tôle ondulée, devenus symboles de prestige.

\subsection{Djenné: grandeur et modernité d'un patrimoine historique}

Située au bord du Bani, à $75 \mathrm{~km}$ au sud de Mopti, la ville de Djenné (23 000 hab.) possède un patrimoine architectural exceptionnel à l'échelle de l'Afrique de
l'Ouest. Ses constructions faites de terre crue (banco), héritée d'un passé glorieux pendant lequel la ville jouait le rôle de centre commercial et religieux, sont pourtant très périssables et questionnent la pérennité de la ville.

L'architecture en banco, exigeant un entretien régulier et complet, a beaucoup souffert du déclin économique de la ville consécutif au transfert des activités administratives et commerciales vers Mopti et à la sécheresse des années 1974-1984. Depuis, plusieurs dizaines de maisons sont tombées en ruine, illustrant le fait que 
l'état dans lequel se trouve l'architecture est le baromètre des problèmes économiques auxquelles une grande partie de la population urbaine est confrontée (chute des revenus, nécessité de l'émigration). Comme ailleurs en Afrique Occidentale, la modernisation des habitations et le changement de composition des familles expliquent que la distribution spatiale des anciennes maisons ne correspond plus aux exigences actuelles. Cette modernisation peut prendre la forme d'un changement du plan de base, des surfaces habitables ou du type de façade ou conduire à un remplacement des matériaux originaux. Des matériaux et des techniques modernes ayant l'avantage d'exiger moins d'entretien que le banco gagnent la faveur des habitants au gré des rénovations. Compte tenu de la pauvreté locale, tous les citadins ne peuvent pas (encore) se permettre de construire en matériaux durs, il devient, cependant, évident que la ville ne correspond plus guère à son état d'origine et que cette évolution pourrait questionner non seulement son inscription au Patrimoine de l'Humanité mais également son intérêt touristique.

Pour faire face à ce défi, les Pays-Bas ont joué depuis les années 1970 un rôle important dans l'approfondissement de la connaissance de la ville de Djenné et ses projets de conservation. Au cours d'une mission d'inventaire en 1995, les données globales des maisons furent comparées à celles rassemblées par VAN RANGELROOY (1984). L'inventaire montrait clairement qu'à cette date, la situation était devenue catastrophique: sur les 134 maisons du plan des années 1980, 40 avaient disparu et 34 nouvelles habitations avaient été construites suite à la destruction d'anciens édifices (BEDAux, Diaby \& MAAs 2003). La législation en vigueur n'était que rarement respectée: bien que la ville soit classée et qu'il soit nécessaire pour toute modification d'obtenir au préalable une autorisation, les propriétaires des maisons les détruisaient et les reconstruisaient à leur gré. Les autorités maliennes ont alors pris l'initiative d'établir une Mission Culturelle chargée de la sensibilisation et du contrôle in situ (photo 1).

La situation n'était toutefois pas désespérée. Grâce au savoir-faire des maçons locaux, une continuité architecturale a été préservée. En tant que créateurs et bâtisseurs, ces maçons transmettent la technique spéciale de la construction en terre et il était donc d'une importance primordiale d'impliquer leur corporation dans l'élaboration d'un projet de conservation et de prendre en considération leurs techniques et leurs matériaux. Dans un premier temps, le choix des maisons à restaurer s'est porté sur les maisons à façade monumentale en raison de leur authenticité (GARDI, MaAs \& Mommersteeg 1995). Ceci a été complété par la restauration des ensembles architecturaux situés autour des places publiques et de certaines maisons monumentales individuelles. La restauration s'est basée sur le respect strict des principes de la construction traditionnelle, notamment le crépissage bisannuel. Au cours de ces initiatives, l'organisation des maçons a été revitalisée et a pris une part toujours plus active, ce qui a permis d'augmenter la durabilité du projet. Les maçons peuvent maintenant, avant le début des travaux, établir un devis raisonnable et comprendre les principes de restauration, des aptitudes techniques d'une importance primordiale pour l'avenir de cette catégorie professionnelle.

Suite à l'achèvement formel en 2003 de la première phase du projet, le Ministre de la Culture du Mali s'est engagé à verser une subvention annuelle à la Mission Culturelle en faveur de l'entretien des monuments restaurés, complétée par la commune de Djenné (taxe touristique locale ou subvention du Ministère du Tourisme) et peut-être par les propriétaires des monuments. Les résultats encourageants de la première phase du projet de conservation ont encouragé les Pays-Bas à poursuivre leurs activités au sein de la ville de Djenné, notamment à travers la restauration de maisons monumentales, de sept ensembles n'ayant pas figuré dans la première phase et en une révision d'entretien pour les autres maisons de ces ensembles. Ces initiatives ont permis d'améliorer l'offre touristique de la ville, aujourd'hui au programme de la plupart des agences voyageant au Mali.

\subsection{Falaises de Bandiagara-Pays Dogon: le tourisme au secours de la culture?}

Situé à l'est du Delta intérieur du fleuve Niger, le Pays Dogon est, depuis 1989, partiellement classé au Patrimoine de l'Humanité au double plan naturel et culturel. Les villages dogon nichés dans les éboulis de la Falaise ou sur le plateau présentent une grande diversité architecturale, marquée par l'utilisation de matériaux très divers (BEDAUX \& VAN DER WALLS 2004). Ceci complique la conservation qui est mise en œuvre, car il ne suffit pas de concentrer l'attention sur un nombre limité de sites. En outre, la sauvegarde de ce patrimoine architectural est, de manière croissante, menacée par l'abandon des sites les plus anciens au profit de la plaine, consécutive à la difficulté d'accès des villages perchés, à leur approvisionnement fastidieux en eau et à la disparition des menaces nomades peul et mossi qui avaient justifié l'installation initiale.

Dans ces conditions, des travaux ont été entrepris au sein d'un projet financé par les Pays-Bas pour la sauvegarde de ce patrimoine architectural. Deux méthodes ont été appliquées: la restauration des sites abandonnés depuis plusieurs années et la conservation des sites risquant d'être abandonné.

La première méthode a été appliquée à l'ancien village de Teli. Abandonné il y a une quinzaine d'année, 




Photo 1:Vue des anciens quartiers de la ville de Djenné montrant l'usage prédominant de la brique de terre crue (banco) dans la construction traditionnelle Old neighbourhoods in Djenné built in traditional manner using mud bricks («banco»)

Alte Quartiere von Djenné in traditioneller Lehmziegelbauweise («banco»)

Photo: P. Lemineur, 2005

sa situation au pied de la Falaise de Bandiagara l'a préservé d'une destruction complète. Dans ce cas précis, les villageois avaient choisi d'habiter en hauteur pour dominer la plaine et voir venir leurs ennemis esclavagistes. La disparition de cette menace suite à la colonisation, le manque de place et une vie plus facile dans la plaine ont donné lieu à une migration vers le bas. L'arrivée de la religion musulmane a probablement aussi accéléré cette migration. L'ancien village est remarquable par ses petits lieux d'habitation parfois étagés et construits en pierre. Seuls les greniers, composés de fines parois érigées sur une base en bois, sont construits en banco. Les habitants installés dans le nouveau village les utilisent toujours pour garder le surplus de leurs récoltes. Le fait que certains sanctuaires soient encore fréquentés prouve également l'importance de ces anciens sites pour la communauté dogon actuelle. La méthode adoptée est partie du principe que seule une restauration participative permettait de pérenniser la sauvegarder du site. Etant donné que les édifices sont érigés par des membres des familles et non par une corporation de bâtisseurs comme à Djenné, il est plus difficile d'y introduire une notion de restauration. Ce qui veut dire qu'il a été nécessaire de sauvegarder les éléments architecturaux les plus anciens et de reconstruire uniquement les éléments dont l'existence a été certifiée par la tradition orale. Les villageois euxmêmes avaient déjà entamé des activités de reconstruction, dans le but de faciliter l'accès des touristes et avaient conçu de nouvelles décorations pour rendre leur ancien site plus attractif aux yeux des visiteurs (photo 2).

La deuxième méthode a été appliquée par le projet néerlandais sur le village de Bolimba, situé dans la région du Piniari, à l'ouest de Bandiagara. Elle consiste 

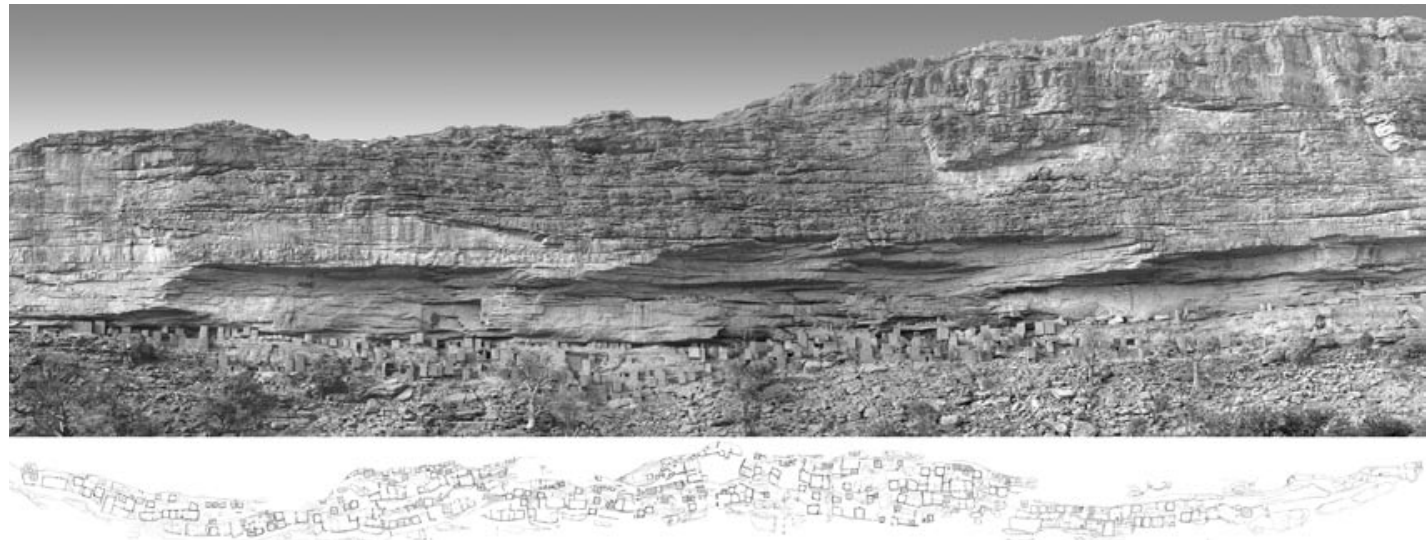

Photo 2: L'ancien village de Teli niché dans la Falaise de Bandiagara. Le dessin montre l'emplacement des constructions anciennes.

Old village of Teli in the Bandiagara Escarpment. The drawing shows the location of traditional buildings. Das alte Dorf Teli in der Bandiagara-Felswand. Die Zeichnung zeigt die Position der traditionellen Häuser. Photo: P. Lemineur, 2005

à prendre des mesures préventives avant l'abandon des villages et garantir la conservation du patrimoine architectural pour les générations futures. Ceci permet aux chercheurs d'analyser ces sites exceptionnels, caractérisés comme à Bolimba par une architecture de forteresse typique de certains villages du Piniari. Les habitations se composent d'un ensemble de cylindres de différentes hauteurs et de plusieurs étages. Les lieux d'habitation, constitués de la cuisine, des chambres à coucher et des étables, y sont très sombres au rez-de-chaussée alors que les niveaux supérieures sont occupés par des greniers. Le volume se termine par un couvercle en pierre ou par un accès donnant sur le toit où l'on sèche les aliments.

Dans ces sites dogon, le tourisme est organisé dans ce que les anthropologues appellent une bulle touristique, un environnement protégé dans lequel les relations entre touristes et acteurs locaux s'entremêlent sans se détruire. A l'indépendance du pays en 1960 en effet, l'exploitation touristique était gérée de façon étatique, les flux touristiques étant dirigés vers Sangha et ses villages proches. Dans les années 1980, les ajustements structurels ont conduit à une libéralisation du marché, avec pour résultat de multiplier hôtels, campements, agences de voyages, guides officiels et officieux, commerces d'«antiquités» et troupes de danse. La bulle touristique étatique s'est alors rapidement diversifiée, employant des Dogon et des Maliens d'autres ethnies, tandis que l'ensemble des villages de la falaise ainsi que de nombreuses localités du plateau et de la plaine étaient graduellement concernés par le tourisme.

L'impact d'un tel phénomène est normalement considéré avec appréhension par les agences de voyage, qui décrivent leurs destinations comme immaculées et intactes et qui considèrent qu'un tourisme intensif détruit une destination exotique ou une culture locale. A ce titre, le Pays Dogon est considéré par la littérature de voyage comme l'exemple ultime d'une culture africaine ayant résisté aux influences occidentales et coloniales. Bien avant sa classification par l'UNESCO, les travaux des ethnologues français ont contribué à l'«exotisation» de la culture dogon (CIARCIA 2003). Tout en étant sévèrement critiquée (VAN BeEK 1991, 2004), cette littérature ethnographique a nourri le projet touristique dogon d'histoires racontant des pratiques et des connaissances indigènes aussi insoupçonnées qu'exotiques.

La réalité sur le terrain est cependant différente. L'impact du tourisme n'est guère synonyme de catastrophe et la vie quotidienne des Dogon se déroule bien souvent sans aucune référence au tourisme. Naturellement, les visiteurs génèrent des revenus (VAN BEEK 2006), mais les touristes sont une «réalité alternative» (URRY 2002), vivant dans leur «bulle» et fréquentant les villages par des voies prédéterminées et canalisées. L'anthropologie touristique a ainsi reconnu que la 
bulle touristique, c'est-à-dire les services entourant le touriste dans le terrain étranger, possède deux fonctions: elle sépare non seulement le touriste de la réalité brute du pays qu'il visite, en facilitant une «aventure confortable», mais également en dissociant le village de l'impact de la présence des étrangers, en déléguant le contact avec les visiteurs à des professionnels.

Ceci concerne également les arts: sous la pression du tourisme, la fabrication et la vente des objets culturels, comme les portes, les masques, les statuettes ou les serrures ont connu une véritable explosion. Bien que souvent méprisés par les historiens de l'art africain, ces objets rappellent le style dogon et sont fabriqués avec des outils traditionnels. Ils peuvent être plus petits, afin d'entrer dans la valise du touriste, et fabriqués en grande quantité, mais ils représentent tout de même une production culturelle considérable et inventive. Ce phénomène illustre d'une part la continuité d'une tradition, qui connaît le changement comme n'importe quelle tradition, et d'autre part l'invention d'une tradition, ce qui explique que ces objets relèvent à la fois de l'authentique et d'une authenticité inventée. Les danses des masques, très recherchées par les touristes, ont les mêmes caractéristiques. Une des danses exécutée pour les touristes a ainsi été créée à partir d'un fragment de la cérémonie de deuil (dama) (VAN BEEK 2001). Cette danse, qui obéit normalement à des codes culturels précis, est aujourd'hui isolée de son contexte culturel, raccourcie et exécutée à la commande en dehors de la place de danse du village. Cet exemple montre que la performance culturelle touristique correspond bien à certaines affinités des Dogon pour la danse, mais est devenue un élément d'échange, une revendication de l'authenticité. La demande continue entretenue par le tourisme permet d'envisager que de nouveaux éléments culturels soient progressivement intégrés au spectacle touristique (VAN BEEK 2006).

\subsection{Potentialités et contraintes touristiques des Monts Hombori}

L'inaccessibilité relative des Monts Hombori (1155 m), situés à $300 \mathrm{~km}$ à l'est de Mopti, en a fait une destination reconnue parmi les alpinistes, de même qu'un haut lieu de la biodiversité mondiale (IosET et al.2004). Un millier de nuitées annuelles attestent aujourd'hui de l'engouement des touristes pour ce lieu dont la richesse culturelle est attestée par un habitat défensif perché présentant de fortes similitudes avec celui du Pays Dogon. Bien que le patrimoine naturel et culturel de Hombori puisse être considéré comme exceptionnel à l'échelle de l'Afrique de l'Ouest, aucune mesure de protection ou de valorisation n'a encore vu le jour dans la région.

Dans ce contexte, les revenus issus du tourisme demeurent extrêmement faibles. La ville de Hom- bori est l'unique endroit où la vente d'objets d'artisanat, les services hôteliers et les activités de guidage assurent quelques gains touristiques. Dans les autres villages, les touristes ne consomment ni produits artisanaux, ni biens de consommation, ni services spécialisés comme c'est l'usage dans la Falaise de Bandiagara. En termes de conservation du patrimoine naturel et construit, il s'agit assurément d'un avantage qui «préserve les traditions» et les écosystèmes d'une transformation brutale et mercantile. Cependant, il est tout aussi manifeste que l'absence de revenus issus du tourisme crée une situation conflictuelle qui ne peut déboucher sur des perspectives de développement satisfaisantes. En effet, le maintien de ce qui fait la richesse culturelle et écologique des lieux est aujourd'hui remis en question par la paupérisation généralisée des populations.

Du fait de l'urgence de certaines préoccupations liées à l'accès à l'eau ou au marché du travail qui reflètent le problème plus général de l'enclavement de ces territoires, la question des apports touristiques ne constitue pas toujours une priorité pour les populations des sites perchés. Celles-ci revendiquent avant tout les moyens d'accéder à de meilleures conditions de vie. Cependant, dans l'état actuel des finances communales, il n'est pas certain que cette question soit réellement à l'ordre du jour. Il est toutefois bien certain que le tourisme pourrait servir d'accélérateur du développement local, si une stratégie concertée parvient à lier les problématiques concernant l'amélioration des conditions matérielles de vie dans les sites perchés au phénomène touristique. L'analyse du contexte montre qu'il est possible à très court terme d'envisager un développement selon trois axes principaux: création d'infrastructures d'hébergement et de services touristiques, amélioration de l'accessibilité aux sites perchés et de la desserte en eau:

- L'organisation actuelle pourrait être améliorée en créant de petits relais touristiques rudimentaires. L'exemple du Pays Dogon montre que certains individus au profil atypique (chrétiens, particuliers disposant d'un capital de départ, fils cadets de chefs de village, jeunes ambitieux revenus de l'émigration) fonctionnent comme des pionniers du tourisme et y introduisent progressivement des services spécifiques (Walther 2003). Par conséquent, la diversification de l'offre touristique en matière d'hébergement et de commodités ne se heurte pas seulement à des obstacles liés au niveau de l'investissement, lequel dépasserait les capacités locales de financement, mais également à l'absence d'individus entreprenants, qui vont approvisionner les villages en boissons, installer de petits campements dans leur concession et aménager des boutiques d'artisanat.

- La desserte des villages des Monts Hombori est actuellement une entrave importante à la création de structures d'hébergement et, plus généralement, 
à la fréquentation des visiteurs. Dans ces conditions, l'aménagement de sentiers pédestres liant les sites entre eux constitue une priorité. De l'avis des guides locaux, plusieurs itinéraires pourraient être réhabilités ou créés pour permettre de multiplier les possibilités de randonnées. Ces aménagements pourraient être réalisés grâce à des chantiers à haute intensité de main-d'œuvre.

- L'accès à des ressources hydrauliques plus abondantes et plus régulières constitue la préoccupation de toutes les populations localisées en altitude. Aujourd'hui, l'eau provient des forages situés dans la plaine et, de manière saisonnière, de sources nichées dans les éboulis ou les falaises. Au vu de ces situations et dans l'hypothèse où le maintien des populations perchées est une condition du développement touristique, la dimension hydraulique doit constituer un élément central de tout projet touristique, tant la disponibilité en eau pénalise les conditions de vie des populations vivant en altitude (photo 3).

Le développement de l'offre de services se heurte à plusieurs contraintes, au nombre desquelles figurent les interdits religieux qui s'appliquent à certains lieux de culte situés dans les massifs, de même que les dynamiques conflictuelles qui animent les pouvoirs locaux et qui sont, dans certains cas, susceptibles de freiner les initiatives touristiques:

- La diversification de l'offre touristique est limitée par la réticence des villageois qui craignent que le tourisme ne porte atteinte aux nombreux endroits sacrés situés dans la région. Depuis que l'islam est devenu socialement dominant dans les Monts Hombori (env. 1950), la fréquence et l'intensité des manifestations animistes qui étaient directement liées aux Monts Hombori a singulièrement diminué. Dans cette perspective, les sommets les plus problématiques du point de vue de la fréquentation touristique sont ceux qui appartiennent aux villages demeurés fidèles à l'animisme. Les alpinistes qui essaient d'emprunter les parois où se nichent ces objets sacrés encourent la colère légitime des populations. Les villageois refusent également de laisser les touristes monter seuls et militent pour que les étrangers soient accompagnés dans leur ascension pour éviter que des visiteurs non avertis se rendent dans des zones interdites. Ce risque plaide en faveur d'un encadrement local du tourisme par des guides qui soient spécifiquement formés et sachent reconnaître la valeur symbolique des lieux.

- Le tourisme apparaît certes comme un facteur de domination car les inégalités de revenus et de statut sont très largement favorables aux visiteurs étrangers. Cependant, il peut également servir à la création de nouveaux rapports de force entre ceux qui sont intégrés dans la bulle touristique et ceux qui vivent en marge. C'est pourquoi une stratégie de mise en tourisme des lieux ne peut faire l'économie de l'analyse des affrontements politiques qui animent la scène locale. A Hombori, ceux-ci semblent relativement prononcés du fait du caractère «insulaire» du site et de la longue tradition de résistance de ses habitants vis-à-vis d'envahisseurs très divers. L'enjeu de la chefferie ou du pouvoir politique communal reste central et les rivalités qui marquent le contexte local opposent généralement les membres des familles de la chefferie entre eux, de même que ces membres à d'autres prétendants. Ces rivalités ont des conséquences sur la dynamique des projets touristiques, parce qu'elles focalisent l'attention et les ressources sur des conflits politiques et généalogiques au lieu de se concentrer sur les problèmes socio-économiques.

\section{Conclusion: protection du patrimoine et développement local}

Au terme de l'analyse, il devient évident que les initiatives de protection et de valorisation du patrimoine entreprises au Mali dépendent très largement de chaque contexte d'intervention mais que, d'une manière générale, ces dynamiques ne peuvent se dispenser de viser à l'amélioration des conditions de vie des populations. A Djenné, les profondes transformations socio-économiques de la ville ont entraîné une dégradation rapide du patrimoine construit en banco. Les initiatives de conservation ont alors tenté de préserver les marques d'un passé révolu quoique prestigieux, dans un contexte de modernisation de l'habitat urbain qui rend toute muséification architecturale impossible. Dans la Falaise de BandiagaraPays Dogon et à Hombori, la difficulté de préserver ce patrimoine est rendue plus ardue par le fait qu'un certain nombre de villageois ont décidé de se déplacer en plaine de façon plus ou moins définitive en délaissant leur habitat traditionnel. Si des travaux de revalorisation ont été entrepris dans la Falaise, grâce à son inscription au Patrimoine de l'Humanité, aucune initiative de conservation n'est à ce jour entreprise à Hombori, alors que ses qualités écologiques et culturelles pourraient le justifier.

Il demeure que ces attributs hérités - architecture de banco de Djenné et sites d'altitude - constituent aujourd'hui l'argument le plus déterminant des stratégies touristiques, conjuguant culture, découverte et trekking. Les endroits les plus susceptibles de retenir l'intérêt des visiteurs sont paradoxalement ceux qui abritent encore des habitants «authentiques» du point de vue des agences et des touristes, c'est-à-dire qui conservent une vie sociale peu altérée par la mise en tourisme des lieux. Dès lors, les stratégies touris- 


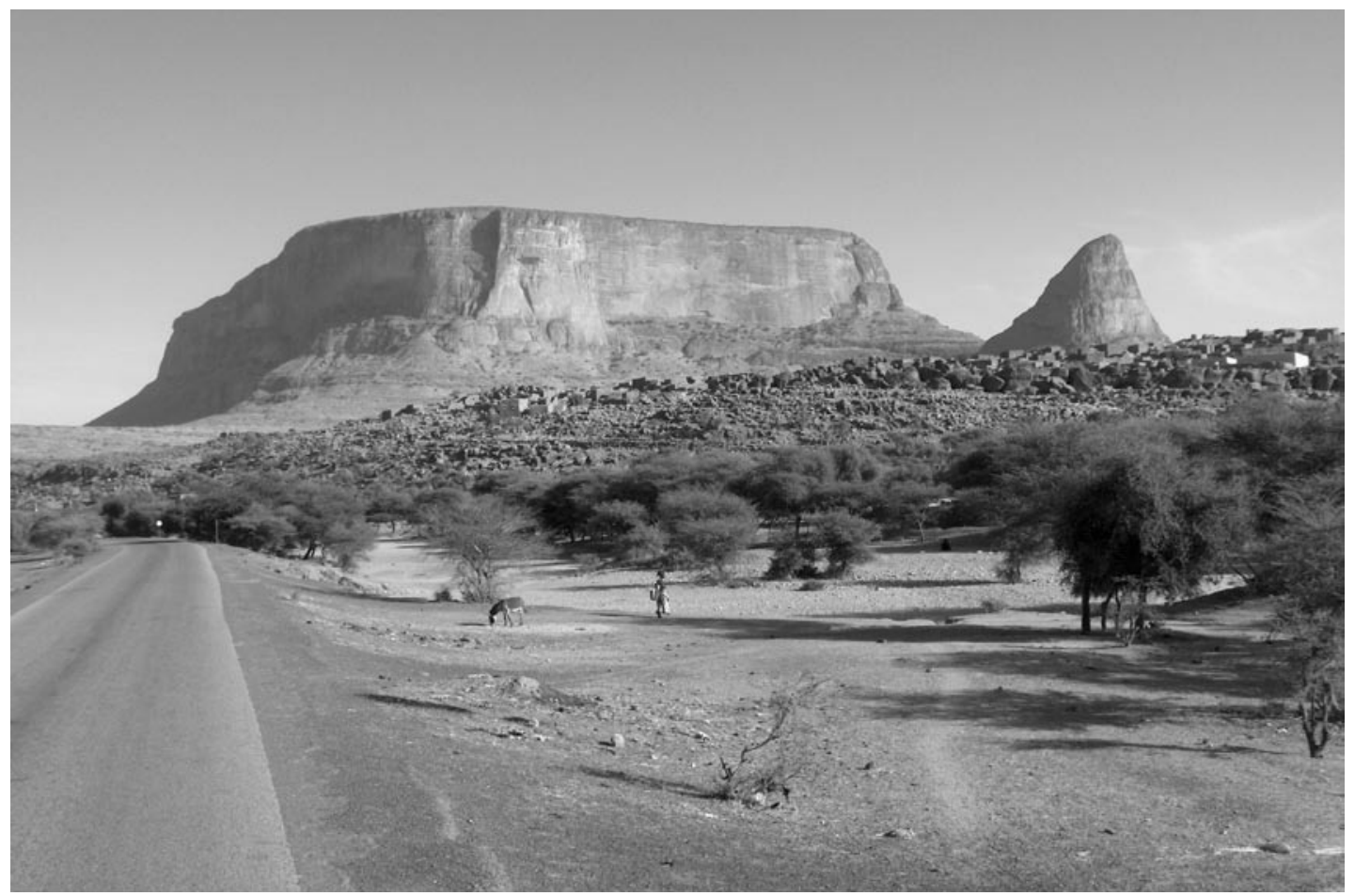

Photo 3: Le Mont et la ville de Hombori (Mali): l'habitat perché défensif est une caractéristique du peuplement de la région.

Mount Hombori and Hombori City (Mali): the raised position of defence is typical for the area.

Der Berg und die Stadt Hombori (Mali): die erhöhte Verteidigungsposition ist ein Charakteristikum für die Bevölkerung der Region.

Photo: O. WALTHER, 2005

tiques mises en place dans les sites de Djenné, du Pays Dogon et de Hombori devraient partager une propriété commune: permettre la survie de ces sites privilégiés à travers l'amélioration des conditions de vie de la population. Compte tenu de la dégradation des conditions climatiques générales en Afrique sahélienne, le tourisme devrait être considéré comme une alternative économique aux secteurs traditionnels de l'élevage et de l'agriculture céréalière.

Disposant d'une longueur d'avance sur les autres sites, le Pays Dogon préfigure peut-être l'évolution du tourisme malien, marqué par une bulle touristique indispensable à l'exercice des activités quotidiennes en marge des initiatives touristiques. Là où existent des programmes de conservation du patrimoine, la fréquentation touristique peut alors se concevoir comme une activité doublement profitable, dans la mesure où elle offre des gains alternatifs et permet, dans une cer- taine mesure, de participer à l'entretien d'une tradition renouvelée par le contact avec l'extérieur. Dans les situations où, comme à Hombori, les gains du tourisme sont presque inexistants, l'accessibilité des sites perchés devrait être améliorée par la réalisation d'ouvrages hydrauliques capables de répondre durablement à l'éloignement et à la raréfaction des sources d'approvisionnement et par la mise en place de chemins d'accès plus aisés.

Il découle de ces exemples que le classement d'un site sur la liste du Patrimoine de l'Humanité doit s'étendre encore davantage à la sauvegarde de la mémoire, du savoir-faire, des valeurs et des coutumes de chaque lieu, c'est-à-dire à la culture qui a produit le cadre matériel que l'on souhaite protéger. La bonne préservation des monuments passe par la prise en compte de divers enjeux, dont celui du développement. Le patrimoine culturel étant l'expression de certaines 
connaissances, valeurs et convictions, il participe de fait à l'identité culturelle du site et de ses habitants. En outre, sachant que ce patrimoine est un bien non renouvelable, il est impératif de réfléchir à la qualité des interventions nouvelles en relation avec les valeurs patrimoniales. La muséification des éléments existants au sein des aires de protection n'est donc pas la meilleure solution. Le débat est celui de la plus-value culturelle, notamment esthétique, sociale et économique que peuvent offrir de nouvelles constructions aux abords des monuments. C'est dans cette perspective, que les aires de protection, justifiées par la présence des monuments, devraient jouer un rôle de zones pilotes pour le développement.

\section{Bibliographie}

Bedaux, R., Diaby, B. \& P. Maas (éds) (2003): L'architecture de Djenné (Mali). La pérennité d'un patrimoine mondial. - Leiden: Rijksmuseum voor Volkenkunde et Gand: Snoeck.

Bedaux, R. \& J.D. van der WaAls (éds) (2004): Regards sur les Dogon du Mali. - Leiden: Rijksmuseum voor Volkenkunde et Gand: Snoeck.

BEek, W.E.A. van (1991): Dogon restudied. A field evaluation of the work of Marcel Griaule. - In: Current Anthropology 32, 2:139-167.

BeEk, W.E.A. van (2001): The Dogon: Africa's people of the cliffs (with photographs by S. Hollyman). - New York: Abrams.

BEEK, W.E.A. vAN (2003): African tourist encounters: effects of tourism on two West African societies. - In: Africa 73, 2: 251-289.

BEEK, W.E.A. vAN (2004): Haunting Griaule: experiences from the restudy of the Dogon. - In: History in Africa 31: 43-68.

BEEK, W.E.A. vAN (2005): Walking wallets? Tourists at the Dogon falaise. - In: Wooten, S. (éd.): Wari matters: ethnographic explorations of money in the Mande world. - Münster: LiT Verlag: 191-216.

BEEK, W.E.A. van (2006): Boys and masks among the Dogon. - In: OtTENBerg, S. \& D.A. Binskley (éds): Playful performers. African children's masquerades. - Londres et New Brunswick: Transaction: 67-88.

Ciarcia, G. (2003): De la mémoire ethnographique. L'exotisme du pays dogon. - Paris : Ed. de l'Ecole des Hautes Etudes en Sciences Sociales (EHESS).

Cissé, L. (1995): Les missions culturelles au Mali: genèse et principaux objectifs. - In: WAMP Bulletin 6: 110-111.

DiJK, D., Lemineur, P. \& I. Schuts (2004): La construction de l'espace. Espaces et habitats dans la région de la Falaise de Bandiagara. - In: Bedaux, R. \& J.D. van DER WaAls (éds): Regards sur les Dogon du Mali. - Leiden: Rijksmuseum voor Volkenkunde et Gand: Snoeck: 68-72.

Gardi, B., MaAs, P. \& G. Mommersteeg (1995):
Djenné, il y a cent ans. - Amsterdam: Institut Royal des Tropiques.

Ioset, J.-R., Renaud, T., Diallo, D. \& J. Kissling (2004): Biodiversity monitoring, protection and integrated development of the Mount Hombori region. - In: ISE Newsletter 4, 2: 7-8.

JHA, S. (2005): Can natural world heritage sites promote development and social harmony? - In: Biodiversity and conservation 14: 981-991.

Ouallet, A. (2002): Patrimoine mondial et pauvreté locale. - In: Annales de la recherche urbaine 92: 87-94. Rangelrooy, A. van (1984): Toubabou, ça va? Een architectuur onderzoek in Djenné, Mali. - Eindhoven: T.U. Eindhoven.

UNESCO (2006): Le Patrimoine mondial. - www.whc. unesco.org 15.5.2006.

URRY, J. (2002): The tourist gaze. - London: Sage.

WALther, O. (2003): Le tourisme au Pays Dogon entre verticalité et répétition de la tradition. - In: REYNARD, E., Holzmann, C., Guex, D. \& N. Summermatter (éds): Géomorphologie et tourisme. Actes de la réunion annuelle de la Société suisse de géomorphologie (SSGm), Finhaut, 21-23 septembre 2001. - Lausanne: Travaux et recherches de l'Institut de Géographie de l'Université de Lausanne 24: 93-104.

Walther, O. (2006): Le développement du tourisme dans les Monts Hombori. - Lausanne: Institut de Géographie et Neuchâtel: Projet de monitoring écologique HOMBORI, www.hombori.org 20.5.2006.

\section{Résumé: Tourisme et patrimoine au Mali. Destruction des valeurs anciennes ou valorisation concertée?}

Cet article traite des rapports conflictuels entre tourisme et patrimoine au Mali. En prenant pour exemple les sites de Djenné, des Falaises de Bandiagara-Pays Dogon et de Hombori, il étudie plus précisément la mise en tourisme de ces lieux, qui attirent un nombre croissant de visiteurs grâce à un patrimoine naturel et culturel exceptionnel, ainsi que les initiatives de conservation qui ont été entreprises dans un cadre international. Ce faisant, il postule que le tourisme peut constituer un facteur de développement pour les populations qui habitent les sites concernés sous réserve d'une politique de promotion concertée, alliant conservation et valorisation.

\section{Abstract: Tourism and heritage in Mali. Erosion of traditional values or targeted enhancement of value?}

This article deals with the sensitive relationship between tourism and heritage protection in Mali. Focusing on the areas of Djenné, the Cliffs of Bandiagara in Dogon country and Hombori, developments within the booming tourism industry are analysed. This boom in particular reflects the exceptional natural and cultural heritage of these places. It has also profited from the conservation initiatives that have 
been undertaken at an international level. It is argued that tourism can contribute towards improving the standard of living of the inhabitants of these famous spots, provided that a promotion policy is put in place that combines conservation and appreciation with development.

Zusammenfassung: Touristische Entwicklung und der Schutz von Kulturgütern in Mali - Zerstörung traditioneller Werte oder konzertierte Wertsteigerung?

Dieser Artikel behandelt die konfliktbeladenen Beziehungen zwischen touristischer Entwicklung und dem Schutz von Kulturgütern in Mali. Am Beispiel von Djenné, der Bandiagara-Felswand im Gebiet der Dogon und von Hombori wird die Entwicklung touristischer Einrichtungen beleuchtet, die dank eines einzigartigen Natur- und Kulturerbes eine wachsende Anzahl von Besuchern anziehen. Gleichzeitig werden auch die durch internationale Bemühungen erfolgten Massnahmen zur Erhaltung des Kulturerbes dargestellt. Die Bedeutung des Tourismus als möglicher Faktor für die Verbesserung der allgemeinen Lebensbedingungen der örtlichen Bevölkerung wird unterstrichen. Voraussetzung dazu ist ein Vermarktungskonzept, welches Bewahrung und Wertschätzung gleichermassen einbindet.

Prof. Dr. Walter E.A. van Beek, African Studies Centre, Leiden \& Department of Cultural Anthropology, University of Utrecht, K. 1222, P.O. Box 80.140, 3508 TC Utrecht, The Netherlands.

e-mail:W.E.A.vanBeek@fss.uu.nl

Dipl.-arch. Philippe Lemineur, ORIGIN Architecture

\& Engineering sprl, Rue des Chartreux 17, B-1000 Bruxelles, Belgique.

e-mail:philippe.lemineur@origin.eu

Dr. Olivier Walther, Département Géographie et Développement (GEODE), Centre de Populations, de Pauvreté et de Politiques Socio-Economiques (CEPS/ INSTEAD), BP 48, L-4501 Differdange, Luxembourg. e-mail: Olivier.Walther@ceps.lu

\section{Manuskripteingang/received/manuscrit entré le}

18.5.2007

Annahme zum Druck/accepted for publication/accepté pour l'impression: 4.12.2007 